\title{
A Study of the Role of the Hexose Monophosphate Pathway with Respect to Fatty Acid Biosynthesis in Sporulation of Saccharomyces cerevisiae
}

\author{
By J. RICHARD DICKINSON ${ }^{*}$ AND MICHAEL J. E. HEWLINS ${ }^{2}$ \\ ${ }^{1}$ Department of Microbiology, University College, Cardiff CF2 ITA, UK \\ ${ }^{2}$ Department of Chemistry, University College, Cardiff CFI IXL,UK
}

(Received 2 June 1987; revised 16 September 1987)

\begin{abstract}
${ }^{13} \mathrm{C}$ NMR was used to study the pattern of label incorporation from $\left[2-{ }^{13} \mathrm{C}\right]$ acetate into trehalose during sporulation in Saccharomyces cerevisiae. A wild-type strain and a strain homozygous for the $z$ wf I mutation (which affects glucose-6-phosphate dehydrogenase) were used. In the wildtype it was possible to deduce the cycling of glucose 6-phosphate around the hexose monophosphate pathway whilst in the mutant strain this did not occur. The requirement of the hexose monophosphate pathway for providing NADPH for fatty acid biosynthesis was examined using ${ }^{13} \mathrm{C}$ NMR and GC/MS. The wild-type strain produced a typical profile of fatty acids with palmitoleic acid being the most abundant whereas the mutant contained only onequarter the amount of total fatty acid. As $z w f l$ homozygous diploids are able to sporulate this indicates that the large amount of fatty acid biosynthesis observed in sporulation of wild-type strains is not essential to the process.
\end{abstract}

\section{INTRODUCTION}

Diploid strains of Saccharomyces cerevisiae can undergo a developmental switch from vegetative growth and mitosis to the alternative processes of meiosis and ascospore formation. This switch can only take place in strains which are heterozygous at the mating-type locus, can only occur during the Gl phase of the cell cycle, and is regulated by nutritional conditions (Esposito \& Klapholz, 1981). Sporulation can be induced efficiently by transferring cells from a rich growth medium into one containing a poorly utilized carbon source such as acetate, and in the absence of nitrogen, for $\mathrm{NH}_{4}^{+}$in particular is a very strong repressor of sporulation (Esposito \& Klapholz, 1981).

Previous work aimed at characterizing the metabolism of sporulation showed that initiation of sporulation involves a rapid change in the operation of the tricarboxylic acid and glyoxylate cycles such that the intracellular concentration of glutamate increases (Dickinson et al., 1983, 1985). At about $4 \mathrm{~h}$, net glutamate synthesis ceases and trehalose synthesis is initiated via glyoxalate cycle derived intermediates. There is an absolute requirement for an intact sequence of gluconeogenic enzymes to accomplish this phase of the process (Dickinson \& Williams, 1986). Subsequently fatty acid synthesis begins, concomitant with onset of operation of the hexose monophosphate pathway (Dickinson et al., 1983). We reasoned that the apparent coupling of fatty acid biosynthesis with the involvement of the hexose monophosphate pathway is more than fortuitous coincidence. The hexose monophosphate pathway gives rise to NADPH which is required in the reductive biosynthesis of fatty acids. In order to test our hypothesis about the role of the hexose monophosphate pathway in relation to fatty acid biosynthesis we studied a wild-type diploid strain and a homozygous diploid affected in the ZWFI gene [glucose-6phosphate dehydrogenase, the first enzyme of the oxidative portion of the hexose monophosphate pathway (Lobo \& Maitra, 1982)]. 


\section{METHODS}

Strains, media and cultural conditions. The wild-type strain S41 of Saccharomyces cerevisiae $\left(\frac{M A T \mathrm{a}}{M A T \alpha} \frac{H O}{H O} \frac{\arg 4-1}{\arg 4-1} \frac{c y h l}{c y h l}\right)$ was originally obtained from H. O. Halvorson, Brandeis University, Waltham, Mass., USA. Strain 12.2.1 ( $\left.\frac{M A T a}{M A T \alpha} \frac{\text { metI }}{\text { metl } 1} \frac{A D E 6}{\text { ade6 }} \frac{L E U 1}{l e u l} \frac{z w f I}{z w f 1}\right)$ has been described previously (Dickinson \& Williams, 1986). Cells were grown with shaking at $30^{\circ} \mathrm{C}$ in conical flasks filled to $40 \%$ total volume in a liquid presporulation medium containing $\left(1^{-1}\right) 10 \mathrm{~g}$ yeast extract, $20 \mathrm{~g}$ bacteriological peptone, $20 \mathrm{~g}$ potassium acetate, $1 \mathrm{~g}$ glucose, $0 \cdot 1 \mathrm{~g}$ adenine and $0.1 \mathrm{~g}$ uracil. Before sporulation the cells were grown to a density of $10^{7} \mathrm{cells} \mathrm{m}^{-1}$. Sporulation was induced by the method of Fast (1973). The sporulation medium comprised $1 \%$ (w/v) sodium acetate in $200 \mathrm{~mm}$ potassium chloride containing $150 \mu \mathrm{g}$ benzyl penicillin $\mathrm{ml}^{-1}$. For experiments involving subsequent analysis by ${ }^{13} \mathrm{C}$ NMR, sodium $\left[2-{ }^{13} \mathrm{C}\right.$ ]acetate was used.

Extraction of metabolites. Cells were harvested by filtration using Oxoid 'Nuflow' cellulose acetate membrane filters $(0.45 \mu \mathrm{m}$ grade), washed with distilled water, fixed in ice-cold $5 \%(\mathrm{w} / \mathrm{v})$ perchloric acid and stored deepfrozen overnight. The following day the thawed material was centrifuged for $5 \mathrm{~min}$ at $5000 \mathrm{~g}$. The supernants were carefully neutralized using ice-cold potassium hydroxide and potassium perchlorate was removed by centrifugation. These perchloric acid extracts were used in ${ }^{13} \mathrm{C}$ NMR analyses of the labelling pattern of trehalose (described below). Perchloric acid insoluble material was extracted twice using ethanol/ether $(3: 1, \mathrm{v} / \mathrm{v})$ at $60^{\circ} \mathrm{C}$. This material was frozen in liquid nitrogen and then lyophilized. Prior to fatty acid analysis by ${ }^{13} \mathrm{C}$ NMR these samples were solubilized in $\mathrm{C}^{2} \mathrm{HCl}_{3}$.

To permit analysis of fatty acids by GC/MS methyl esters were prepared. The crude extracted acids were dissolved in aqueous sodium carbonate and washed with ether. The aqueous solution was acidified with hydrochloric acid and then extracted three times with ether. The combined ether extract was evaporated and the residual fatty acids were redissolved in benzene. After addition of methanolic boron trifluoride $(14 \%$, w/v), the mixture was heated under reflux for $15 \mathrm{~min}$ and then cooled to room temperature. Ether was added, followed by aqueous sodium carbonate dropwise with shaking (to remove excess boron trifluoride) until effervescence ceased. The organic layer was removed and the aqueous layer extracted three times with ether. The combined organic layer was dried using anhydrous magnesium sulphate and evaporated to leave the methyl esters. ${ }^{1} \mathrm{H}$ and ${ }^{13} \mathrm{C}$ NMR spectra were recorded.

GC/MS. This was done using a Hewlett-Packard 5970 mass selective detector attached to a Hewlett-Packard 5890 gas chromatograph fitted with a CP-SIL-5 capillary column ( $25 \mathrm{~m}$ length, $25 \mu \mathrm{m}$ diameter). Injection of a $1 \%$ $(w / v)$ ethereal solution of methyl esters was made with injector, detector and transfer line at $250^{\circ} \mathrm{C}$. Column temperature was raised from $60^{\circ} \mathrm{C}$ to $250{ }^{\circ} \mathrm{C}$ at $6{ }^{\circ} \mathrm{C} \mathrm{min}-1$. The individual esters were identified by retention times and mass spectra by comparison with authentic compounds. Quantitative measurement of the various esters was made from the integrated total ion chromatogram.

NMR analyses. ${ }^{13} \mathrm{C}$-labelled trehalose was examined using a Varian XL100 spectrometer operating at $25 \mathrm{MHz}$. Samples were run in $25 \mathrm{~mm}$-potassium phosphate buffer, $\mathrm{pH} 6.0$, containing $30 \%(\mathrm{v} / \mathrm{v})^{2} \mathrm{H}_{2} \mathrm{O} ; 8 \mathrm{~K}$ spectra were obtained using an acquisition time of $0.4 \mathrm{~s}$ and a $10 \mu \mathrm{s}$ pulse width. Continuous high power broad band proton decoupling was applied. Dioxane was used as an internal standard of 67.4 p.p.m.

${ }^{13} \mathrm{C}$-labelled fatty acids were examined using a Bruker WM360 spectrometer operating at $90.5 \mathrm{MHz}$. Samples were run in $\mathrm{C}^{2} \mathrm{HCl}_{3} ; 32 \mathrm{~K}$ data points were obtained over a spectral width of $22000 \mathrm{~Hz}$ with a $30^{\circ}$ pulse angle and a $3 \mathrm{~s}$ delay between pulses. Continuous high power broad band proton decoupling was also used. Tetramethylsilane was used as an internal standard of 0 p.p.m.

\section{RESULTS AND DISCUSSION}

$A{ }^{13} \mathrm{C}$ nucleus will give a single resonance in the NMR spectrum (singlet) unless it is attached to one or more ${ }^{13} \mathrm{C}$ nuclei in which case a multiplet will be observed. Thus labelled $\mathrm{C}-1$ of glucose 6-phosphate will give a singlet if $\mathrm{C}-2$ is unlabelled, a doublet if $\mathrm{C}-2$ is also labelled. The same is true for C-6. Hence comparison of the doublet/singlet ratios for C-1, $1^{\prime}$ and $\mathrm{C}-6,6^{\prime}$ resonances in trehalose of yeast cells sporulating in $\left[2{ }^{13} \mathrm{C}\right]$ acetate reveals the degree of cycling of gluconeogenically synthesized glucose 6-phosphate through the hexose monophosphate pathway (Dickinson et al., 1983). This can be explained as follows: $\left[2-{ }^{13} \mathrm{C}\right]$ acetate entering the cell will give rise to phosphoeno[ $\left[2,3-{ }^{13} \mathrm{C}_{2}\right]$ pyruvate and phosphoenol $\left[1,2,3-{ }^{13} \mathrm{C}_{3}\right]$ pyruvate. The former species would be generated by the glyoxylate cycle alone; it would also be formed along with the 1,2,3-labelled phosphoenolpyruvate from an oxaloacetate pool fully randomized by tricarboxylic acid cycling. It is known that both the tricarboxylic acid cycle and glyoxylate cycle are operating at this time (Dickinson et al., 1983). Gluconeogenesis from 2,3- and $1,2,3-{ }^{13} \mathrm{C}$ - 
Table 1. Double/singlet ratios for $C-6,6^{\prime}$ and $C-1, I^{\prime}$ resonances of $\alpha, \alpha^{\prime}$-trehalose extracted from wild-type and mutant strains

Trehalose was extracted from cells $12 \mathrm{~h}$ after transfer to sporulation medium. A representative set of results is shown.

$\begin{array}{lccc}\text { Strain } & \text { Relevant genotype } & \overbrace{C-6,6^{\prime}}^{\begin{array}{c}\text { Doublet/singlet } \\ \text { ratio }\end{array}} & \mathrm{C}-1,1^{\prime} \\ \text { S41 } & \text { Wild-type } & 2 \cdot 24 & 1.23 \\ 12.2 .1 & \frac{z w f l}{z w f l} & 2.55 & 2 \cdot 24\end{array}$

Table 2. Proportions of fatty acids in the wild-type strain

The fatty acid fraction was extracted from strain S41 $12 \mathrm{~h}$ after transfer to sporulation medium. Fatty acids were converted to the respective methyl esters and then analysed by GC/MS. Quantification was done using the integrated total ion chromatogram. The results are from a single experiment but separate NMR analyses gave good agreement (see text).

\begin{tabular}{lc}
\multicolumn{1}{c}{ Fatty acid } & $\begin{array}{c}\text { Relative abundance of } \\
\text { identified fatty acids } \\
(\%)\end{array}$ \\
Lauric acid $(12: 0)$ & $0 \cdot 81$ \\
Myristic acid $(14: 0)$ & $2 \cdot 78$ \\
Palmitoleic acid $(16: 1)$ & 52.23 \\
Palmitic acid $(16: 0)$ & 24.30 \\
Oleic acid $(18: 1)$ & $16 \cdot 11$ \\
Stearic acid $(18: 0)$ & 3.77
\end{tabular}

labelled phosphoenolpyruvate will give rise to similar isotopic enrichments in both the C-1,2 and C-5,6 portions of the glucose 6-phosphate molecule and hence in trehalose. However, intervention of the hexose monophosphate pathway will result in significant change in the doublet/singlet ratio for $\mathrm{C}-1$ in glucose 6-phosphate (and hence trehalose) because C-1 and C-2 in glucose 6-phosphate after cycling through the hexose monophosphate pathway are derived from C-2 and C-3 respectively in the original glucose 6-phosphate molecules.

Table 1 shows doublet/singlet ratios for $\mathrm{C}-1,1^{\prime}$ and $\mathrm{C}-6,6^{\prime}$ resonances of trehalose isolated from wild-type and $z w f 1$ homozygous diploid strains $12 \mathrm{~h}$ after transfer to sporulation medium. Doublet/singlet ratios for $\mathrm{C}-6,6^{\prime}$ and $\mathrm{C}-1,1^{\prime}$ resonances of trehalose from the wild-type strain (S41) are different indicating significant cycling of glucose 6-phosphate around the hexose monophosphate pathway. This confirms our earlier observations (Dickinson et al., 1983). However the doublet/singlet ratios for C-6,6' and C-1, $1^{\prime}$ resonances of trehalose from the $z w f$ I homozygous diploid (12.2.1) were virtually identical both to each other and to the figure for C$6,6^{\prime}$ resonance from the wild-type strain. This is the expected result in a mutant lacking glucose 6-phosphate dehydrogenase which is unable to operate a hexose monophosphate pathway and further supports the analytical approach adopted.

Another prediction concerning strain 12.2.1 was that due to its defect in glucose-6-phosphate dehydrogenase it would be much depleted of NADPH as the hexose monophosphate pathway is a major source of NADPH in the cells (Bruinenberg et al., 1983a,b). As a consequence one would also expect this strain to be unable to synthesize the large amounts of fatty acids that are produced in sporulation. Table 2 shows the relative proportions of fatty acids in the wild-type strain S41 $12 \mathrm{~h}$ after transfer to sporulation medium. From the GC/MS analysis it was only possible to identify $97.7 \%$ of the total material. Three other methylated minor components of $M_{\mathrm{r}}$ $208 \mathrm{Da}, 355 \mathrm{Da}$ and $429 \mathrm{Da}$ remain unidentified. Previous work on the fatty acid composition of 
Table 3. Relative amounts of fatty acid in wild-type (S41) and mutant (12.2.1) strains

The fatty acid fraction was extracted from cells $12 \mathrm{~h}$ after transfer to $\left[2-{ }^{13} \mathrm{C}\right]$ acetate sporulation medium and analysed by ${ }^{13} \mathrm{C}$ NMR. The results of two separate experiments are shown.

\begin{tabular}{|c|c|c|c|c|}
\hline \multirow{2}{*}{$\begin{array}{l}\text { Resonance } \\
\text { (p.p.m.) }\end{array}$} & \multirow{2}{*}{$\begin{array}{c}\text { Chemical identity } \\
\text { of resonance }\end{array}$} & \multicolumn{2}{|c|}{$\begin{array}{c}\text { Integral } \\
\text { (arbitrary units) }\end{array}$} & \multirow{2}{*}{$\begin{array}{l}\text { Proportion in } \\
\text { mutant relative } \\
\text { to wild-type ( } \% \text { ) }\end{array}$} \\
\hline & & S41 & 12.2 .1 & \\
\hline $14 \cdot 1$ & $\begin{array}{l}\text { Terminal } \mathrm{CH}_{3} \text { of } \\
\text { all fatty acids }\end{array}$ & $\begin{array}{l}1 \cdot 487 \\
5 \cdot 278\end{array}$ & $\begin{array}{l}0.303 \\
1.804\end{array}$ & $\begin{array}{l}20 \cdot 4 \\
34 \cdot 2\end{array}$ \\
\hline $130 \cdot 0$ & $\begin{array}{l}\mathrm{C}-8 \text { in palmitoleic } \\
\text { acid }\end{array}$ & $\begin{array}{l}0 \cdot 711 \\
4 \cdot 130\end{array}$ & $\begin{array}{l}0 \cdot 073 \\
1 \cdot 331\end{array}$ & $\begin{array}{l}10 \cdot 3 \\
32 \cdot 2\end{array}$ \\
\hline
\end{tabular}

lipids of $S$. cerevisiae strain DCL 740 during sporulation found the proportions of unsaturated and saturated acids were approximately two-thirds and one-third respectively (Illingworth et al., 1973). These results are not greatly dissimilar, though the proportions of the individual fatty acids do vary. This is presumably due to minor differences between strains. Analysis of the methylated fatty acids by ${ }^{1} \mathrm{H}$ and ${ }^{13} \mathrm{C}$ NMR (the latter using both conventional and 'DEPT' techniques), as well as an independent set of experiments to measure fatty acids by ${ }^{13} \mathrm{C} \mathrm{NMR}$ following transfer of cells to $\left[2-{ }^{13} \mathrm{C}\right]$ acetate all served to confirm the results in Table 2. For example, comparison of the intensities of the resonances at 130.5 p.p.m. and 130 p.p.m. (C-10 in oleic acid and C-8 in palmitoleic acid respectively) in S41 $12 \mathrm{~h}$ after transfer to ${ }^{13} \mathrm{C}$-labelled sporulation medium gave a ratio of 0.306 . This agrees well with the ratio of 0.308 calculated from the relative proportions of the same two compounds determined by GC/MS. This shows that the use of $\left[2-{ }^{13} \mathrm{C}\right]$ acetate during sporulation has not failed to label any fatty acids: a potential problem with labelling experiments is that the cell may metabolize material incorporated before exposure to the labelled compound.

A comparison of equivalent resonances in NMR spectra of fatty acid fractions from the two strains showed that strain 12.2.1 contained about one-quarter of the total fatty acid present in the wild-type (Table 3). Although this was the type of result that had been expected its significance is more than mere biochemical 'book-keeping'. These findings indicate that the large amount of fatty acid biosynthesis which occurs during sporulation of wild-type strains of $S$. cerevisiae is not an essential part of that process as $z w f 1$ homozygous diploids are capable of sporulation (Dickinson \& Williams, 1986).

The fatty acid synthesis that does occur in the $z w f 1$ homozygous diploid must be dependent upon other NADPH-yielding reactions such as transhydrogenase, NADP ${ }^{+}$-dependent isocitrate dehydrogenase and/or NADP+-dependent glutamate dehydrogenase. However, other workers have concluded that yeasts do not possess transhydrogenase (Bruinenberg et al., 1983b; van Dijken \& Scheffers, 1986). Also, fatty acid synthesis during sporulation does not begin until net glutamate synthesis has ceased (Dickinson et al., 1983); thus experimental and theoretical (Bruinenberg et al., 1983a) analyses have shown that the contribution of NADP ${ }^{+}$-dependent glutamate dehydrogenase would be negligible. This leaves $\mathrm{NADP}^{+}$-dependent isocitrate dehydrogenase as the only likely source of NADPH for reductive biosynthesis in the glucose-6phosphate dehydrogenase-lacking mutant. Bruinenberg et al. (1983a) have suggested that $\mathrm{NADP}^{+}$-dependent isocitrate dehydrogenase alone is insufficient for total cellular NADPH demand. From this work we might infer that in wild-type strains of $S$. cerevisiae during sporulation, approximately $75 \%$ of fatty acid biosynthesis is dependent upon glucose-6phosphate dehydrogenase and the remaining $25 \%$ upon $\mathrm{NADP}^{+}$-dependent isocitrate dehydrogenase.

We are grateful to R. L. Baxter (University of Edinburgh, UK) and P. K. Maitra (Tata Institute of Fundamental Research, Bombay, India) for helpful discussions and to M. Dowsett and A. S. Williams for skilled technical assistance. 


\section{REFERENCES}

Bruinenberg, P. M., VAN Dijken, J. P. \& SchefFers, W. A. (1983a). A theoretical analysis of NADPH production and consumption in yeasts. Journal of General Microbiology 129, 953-964.

Bruinenberg, P. M., van DiJken, J. P. \& SchefFers, W. A. $(1983 b)$. An enzymic analysis of NADPH production and consumption in Candida utilis. Journal of General Microbiology 129, 965-971.

Dickinson, J. R. \& Williams, A. S. (1986). A genetic and biochemical analysis of the role of gluconeogenesis in sporulation of Saccharomyces cerevisiae. Journal of General Microbiology 132, 2605-2610.

Dickinson, J. R., Dawes, I. W., BoYD, A. S. F. \& BAXTER, R. L. (1983). ${ }^{13}$ C NMR studies of acetate metabolism during sporulation of Saccharomyces cerevisiae. Proceedings of the National Academy of Sciences of the United States of America 80, 58475851.

Dickinson, J. R., Ambler, R. P. \& Dawes, I. W. (1985). Abnormal amino acid metabolism in mutants of Saccharomyces cerevisiae affected in the initiation of sporulation. European Journal of Biochemistry 148, 405-406.

van Dijken, J. P. \& Scheffers, W. A. (1986). Redox balances in the metabolism of sugars by yeasts. FEMS Microbiology Reviews 32, 199-224.

Esposito, R. E. \& KlaPholz, S. (1981). Meiosis and ascospore development. In Molecular Biology of the Yeast Saccharomyces: Life Cycle and Inheritance, pp. 211-287. Edited by J. N. Strathern, E. W. Jones \& J. R. Broach. Cold Spring Harbor, New York: Cold Spring Harbor Laboratory.

FAST, D. (1973). Sporulation synchrony in yeast. Journal of Bacteriology 116, 925-930.

Illingworth, R. F., Rose, A. H. \& Beckett, A. (1973). Changes in the lipid composition and fine structure of Saccharomyces cerevisiae during ascus formation. Journal of Bacteriology 113, 373-386.

Lobo, Z. \& MaITRA, P. K. (1982). Pentose phosphate pathway mutants of yeast. Molecular and General Genetics 185, 367-368. 